Recepción: 06 / 10 / 2016

Aceptación: 27 / 01 / 2017

Publicación: 15 / 05 / 2017

Ciencias económicas y empresariales

Artículo corto

\title{
Propuesta de programa de auditoría financiera para la gestión empresarial en
} Ecuador

Proposal of a financial audit program for business management in Ecuador

Programa da auditoria financeira para a gestão empresarial no Equador

Silvia L. Llamuca Pérez ${ }^{\mathrm{I}}$

silvialorena@gmail.com

Correspondencia: silvialorena@gmail.com

'Doctora en Contabilidad y Auditoria, Magister en Costos y Gestión Financiera, Licenciada en Contabilidad y Auditoría, Contadora Publica, Diplomado Superior en Costos y Gestión Financiera, Docente en la Universidad Tecnológica Indoamérica, Ambato, Ecuador. 


\section{Resumen}

El objetivo de una auditoría, es aumentar el grado de confianza de los usuarios en los estados financieros. Esto se logra mediante la expresión por parte del auditor, una opinión sobre si los estados financieros han sido preparados, en todos los aspectos materiales, de conformidad con un marco de información financiera aplicable. Según la IAASB, una auditoría realizada de conformidad con las NIA y con los requerimientos de ética aplicables, permite al auditor formarse dicha opinión. Se considera entonces que la Auditoría constituye una herramienta de control y supervisión, que contribuye a la creación de una cultura de la disciplina de la organización, y permite descubrir fallas en las estructuras o vulnerabilidades existentes en la organización.

Palabras clave: auditoría financiera; gestión empresarial; Ecuador.

\section{Abstract}

The purpose of an audit is to increase the degree of user confidence in the financial statements. This is achieved through the expression by the auditor of an opinion on whether the financial statements have been prepared, in all material aspects, in accordance with an applicable financial reporting framework. According to the IAASB, an audit conducted in accordance with ISAs and applicable ethical requirements allows the auditor to form such an opinion. It is considered that the Audit constitutes a tool of control and supervision, which contributes to the creation of a culture of the discipline of the organization, and allows to discover flaws in the existing structures or vulnerabilities in the organization.

Keywords: financial audit; business management; Ecuador.

\section{Resumo}

O objetivo de uma auditoria é aumentar o grau de confiança do usuário nas demonstrações financeiras. Isto é conseguido através da expressão pelo auditor, um parecer sobre se as demonstrações contábeis foram elaboradas, em todos os aspectos relevantes, de acordo com uma estrutura de relatório financeiro aplicável. De acordo com o IAASB, uma auditoria conduzida de acordo com as normas de auditoria e requisitos éticos aplicáveis permite que o auditor a formar essa opinião. Então considera-se que a auditoria é uma ferramenta de controle e supervisão, o que 
contribui para a criação de uma cultura de disciplina organizacional e para descobrir falhas nas estruturas ou vulnerabilidades na organização.

Palavras chave: auditoria financeira; gestão empresarial; Equador.

\section{Introducción}

La auditoría, es uno de los procesos que ha tomado un rol importante dentro de los parámetros y fundamentos científicos, está basada en los principios de la contabilidad y en la verificación de los registros, para observar su exactitud; no obstante, éste no es su único objetivo.

El objetivo de una auditoría, es aumentar el grado de confianza de los usuarios en los estados financieros. Esto se logra mediante la expresión por parte del auditor, una opinión acerca de si los estados financieros han sido preparados, en todos los aspectos materiales, de conformidad con un marco de información financiera aplicable. Según la IAASB, una auditoría realizada de conformidad con las NIA y con los requerimientos de ética aplicables permite al auditor formarse dicha opinión.

Se considera entonces, que la Auditoría constituye una herramienta de control y supervisión, que contribuye a la creación de una cultura de la disciplina de la organización, y permite descubrir fallas en las estructuras o vulnerabilidades existentes en la organización. Existen diversos tipos de auditoría, aplicables de acuerdo a las necesidades de los usuarios. Entre estos se encuentran los siguientes: Auditorías de los estados financieros, Auditoría Fiscal, Auditorías Operacionales, Auditoría Forense, Auditorías Administrativas, Auditorías Gubernamentales, etc.

El presente artículo pretende realizar una mirada a los procesos de Auditoría Financiera y su importancia para la gestión empresarial en Ecuador.

\section{Desarrollo}

Existe más de una definición de Auditoría, pero en las diversas conceptualizaciones existen elementos coincidentes que permiten generalizar la idea del proceso de auditoría en general. De esta forma se entiende por Auditoría una recopilación, acumulación y evaluación de evidencia sobre información de una entidad, para determinar e informar el grado de cumplimiento entre la información y los criterios establecidos. 
Se constituye además, en un proceso sistemático para obtener y evaluar de manera objetiva, las evidencias relacionadas con informes sobre actividades económicas y otras situaciones, que tienen una relación directa con las actividades que se desarrollan en una entidad pública o privada.

El fin del proceso, consiste en determinar el grado de precisión del contenido informativo con las evidencias que le dieron origen, así como determinar si dichos informes se han elaborado observando principios establecidos para el caso (Anderser, 1997).

El auditor tiene un papel que desarrollar en este proceso, el cual es, determinar el grado de precisión que existe entre los hechos que ocurren en realidad y los informes que se han elaborado después de haber sucedido tales hechos.

Para el desarrollo del proceso, el auditor cuenta con técnicas y procedimientos específicos. Las técnicas son las herramientas de trabajo del Auditor, y los procedimientos son la combinación que se hace de esas herramientas para un estudio en particular.

Los procedimientos de auditoría, son el conjunto de técnicas de investigación aplicables a una partida o a un grupo de hechos y circunstancias relativas a los estados financieros sujetos a examen, mediante los cuales, el auditor obtiene las bases para fundamentar su opinión.

Debido a que generalmente el auditor no puede obtener el conocimiento que necesita para sustentar su opinión en una sola prueba, es necesario examinar cada partida o conjunto de hechos, mediante varias técnicas de aplicación simultánea o sucesiva.

Por su parte las técnicas de auditoria, son los métodos prácticos de investigación y prueba que el auditor utiliza para comprobar la razonabilidad de la información financiera, que le permita emitir su opinión profesional.

Los procedimientos pueden ser aplicados en cualquier proceso de auditoría, mientras que la elección de las técnicas a usar dependerá del tipo específico de auditoría a realizar (Coopers \& Lybrand, 1997).

La Auditoría Financiera, específicamente se refiere a la revisión de los estados financieros de una empresa o cualquier otra persona jurídica (incluyendo gobiernos) en base a una serie de normas 
previamente establecidas, dando como resultado la publicación de una opinión independiente sobre si los estados financieros son relevantes, precisa, completa y presentada con justicia.

Las auditorias financieras, se suelen llevar a cabo por las empresas, debido al especial conocimiento de información financiera que necesitan. Para su realización efectiva y contable deben regirse por una serie de normas o principios que regulan las auditorias, que son emitidas por las autoridades de los países, conforme a los principios internacionales enunciados por los organismos dirigidos a tal fin.

El proceso que sigue una auditoría financiera, inicia con la expedición de la orden de trabajo y culmina con la emisión del informe respectivo, cubriendo todas las actividades vinculadas con las instrucciones impartidas por la jefatura y relacionadas con el ente examinado.

El primer paso del proceso es la planificación. De esta concepción dependerá la eficiencia y efectividad en el logro de los objetivos propuestos, utilizando los recursos estrictamente necesarios.

Esta fase debe considerar alternativas y seleccionar los métodos y prácticas más apropiadas para realizar las tareas, por tanto esta actividad debe ser cuidadosa, creativa positiva e imaginativa; por lo que necesariamente debe ser ejecutada por los miembros más experimentados del equipo de trabajo.

La planificación de la auditoría financiera, comienza con la obtención de información necesaria para definir la estrategia a emplear y culmina con la definición detallada de las tareas a realizar en la fase de ejecución.

La ejecución del trabajo constituye la segunda fase del proceso de Auditoría Financiera. En esta fase el auditor, debe aplicar los procedimientos establecidos en los programas de auditoría y desarrollar completamente los hallazgos significativos, relacionados con las áreas y componentes considerados como críticos, determinando los atributos de condición, criterio, efecto y causa que motivaron cada desviación o problema identificado.

Es de fundamental importancia que el auditor mantenga una comunicación continua y constante con los funcionarios y empleados responsables durante el examen, con el propósito de 
mantenerles informados sobre las desviaciones detectadas, a fin de que en forma oportuna se presenten los justificativos o se tomen las acciones correctivas pertinentes.

La última fase del proceso de Auditoría Financiera la constituye la comunicación de resultados, sin embargo, ésta se va cumpliendo en el propio transcurso del desarrollo de la auditoría.

Está dirigida a los funcionarios de la entidad examinada, con el propósito de que presenten la información verbal o escrita respecto a los asuntos observados. Comprende también, la redacción y revisión final del informe borrador, el que será elaborado en el transcurso del examen, con el fin de que el último día de trabajo en el campo y previa convocatoria, se comunique los resultados mediante la lectura del borrador del informe a las autoridades y funcionarios responsables de las operaciones examinadas, de conformidad con la ley pertinente.

El informe final redactado debe contener la carta de dictamen que respalda el proceso de auditoría desarrollado, los estados financieros de la empresa auditada, las notas aclaratorias correspondientes a los aspectos básicos que han sido analizados, la información financiera complementaria y por último los comentarios, conclusiones y recomendaciones relativos a los hallazgos de auditoría.

El desarrollo de los procesos de Auditoría Financiera para la gestión empresarial en Ecuador, está determinado por los beneficios y rentabilidades que puede ofrecer la realización de estos procesos en el sector empresarial. Examinar el manejo de los recursos financieros de una empresa, para establecer el grado en que sus servidores administran y utilizan los recursos y si la información financiera es oportuna, útil, adecuada y confiable, puede proporcionarle a la empresa mayor confiabilidad ante los clientes y todos los grupos de interés en sentido general (Spurrier, 2004).

Por otra parte la realización de este proceso, permite además evaluar el cumplimiento de las metas y objetivos establecidos por la empresa, ya sea para la prestación de servicios o la producción de bienes. Permite además verificar que las empresas ejerzan eficientes controles sobre los ingresos públicos.

Otro elemento que permite evidenciar la importancia del proceso de Auditoría Financiera para la gestión empresarial, es que permite verificar el cumplimiento de las disposiciones legales, reglamentarias y normativas aplicables en la ejecución de las actividades desarrolladas por ésta. 
Propicia además el desarrollo de los sistemas de información, como una herramienta para la toma de decisiones y la ejecución de la auditoría, así como la formulación de las recomendaciones dirigidas a mejorar el control interno y contribuir al fortalecimiento de la gestión pública y promover la eficiencia operativa de la empresa.

La gestión es el proceso mediante el cual se obtiene, despliega o utiliza una variedad de recursos básicos para apoyar los objetivos de la organización (Willington, 2005). La gestión empresarial cada día adquiere más importancia, así como el enfoque de la administración estratégica como elemento clave para el éxito de una empresa. Esto ha posibilitado que los procesos de Auditoría Financiera a nivel empresarial vayan ganando cada vez más campo y aceptación en este sector, como herramienta esencial para consolidar la imagen y el posicionamiento de las empresas a nivel internacional.

La Auditoría Financiera es de gran utilidad para las empresas, ya que avala la fiabilidad de los estados financieros, y supone un gran apoyo para la gestión de la empresa contar con un auditor que emita un juicio de valor, con el fin de incrementar la veracidad de sus cuentas y mejorar su control interno.

Luego de realizar el proceso de Auditoría, el auditor puede emitir tres tipos de juicios profesionales: favorable, lo que indica que a su juicio las cuentas representan de forma significativa la imagen fiel de la empresa; con salvedades, cuando el informe tiene esta denominación quiere decir que las cuentas tienen errores, omisiones o limitaciones significativas, pero que no implican incorrecciones generalizadas, en cambio, cuando el resultado es desfavorable, quiere decir que los errores, omisiones o limitaciones encontradas generan incorrecciones generalizadas, de forma que afectan al conjunto de las cuentas (Whittington \& Pany, 2006).

Por último, cabe destacar que el auditor puede denegar la opinión, esto ocurre cuando hay limitaciones de alcance y/o incertidumbres muy significativas, por lo que el auditor no puede obtener la evidencia de auditoría adecuada que le lleve a expresar una opinión.

Sin embargo al auditor, además de dar su opinión sobre los estados contables de la empresa va a ayudar a clarificar la información contable, la cual tendrá mayor fiabilidad ante terceros. También ayudará y aconsejará a la empresa en el tratamiento contable adecuado, por lo que someterse a 
este proceso también sirve como formación al personal encargado de realizar las cuentas. A su vez, realizará recomendaciones, si es preciso, de mejoras en los procedimientos llevados a cabo para fortalecer los controles internos de la entidad.

El auditor, es una ayuda más para la empresa, ya que detectará los errores u omisiones y se los comunicará a la empresa. Además, también diagnosticará posibles situaciones de fraude.

Si los errores son de cierta importancia, el auditor propondrá ajustes y reclasificaciones para subsanarlos, y si la empresa los acepta y los hace suyos, estará presentando unas cuentas anuales correctas y respaldadas por la supervisión y aceptación de un auditor.

\section{Conclusiones}

Las normas de Auditoría en Ecuador, establecen que los procesos sean diseñados y realizados para obtener una certeza razonable de sí, los estados financieros no contienen exposiciones erróneas o inexactas de carácter significativo.

Es de suma importancia, el desarrollo y aplicación de la Auditoría a los estados financieros en las empresas, ya que de esta manera los auditores se comprometen a reunir evidencia y a proporcionar un alto nivel de seguridad para que los estados financieros, cumplan con los principios de contabilidad generalmente aceptados.

\section{Referencias bibliográficas}

Anderser, Arthur. (1997). Diccionario Espasa Economía y Negocios. Madrid: Espasa.

Coopers \& Lybrand (1997). Los Nuevos Conceptos del Control Interno (Informe COSO), Madrid: Espasa-Calpe.

IAASB: (Internacional Auditing and Assurance Standards Board), Objetivo y principios generales que gobiernan una auditoría de estados financieros, NIA 200, 2012.

Whittington \& Pany (2006). Principios de Auditoría, Decimocuarta Edición, McGraw-Hill.

W. Spurrier. (2004). “Análisis Semanal de Economía y política del Ecuador”, Revista de Análisis Semanal, No 4, enero 26. 